\title{
A Domain Decomposition Method for a Geological Crack
}

\author{
O. Bodart ${ }^{1}$, A. Chorfi ${ }^{2}$, and J. Koko ${ }^{2}$
}

\section{Introduction}

The computational cost is a key issue in crack identification or propagation problems. One of the solutions is to avoid re-meshing the domain when the crack moves by using a fictitious domain method [6]. We consider a geological crack in which the sides do not pull apart. To avoid re-meshing, we propose an approach combining the finite element method, the fictitious domain method, and a domain decomposition approach. We first extend artificially the crack to split the domain into two subdomains with a nonpenetration condition (negative relative normal displacement) on the crack, a prescribed homogeneous displacement jump condition (continuous displacement) on the fictitious crack. We obtain a convex linearly constrained minimization problem with a quadratic cost function. We use a (primal-dual) interior points method, see e.g.[3, sect 16.6],[7], for the numerical realization.

The paper is organized as follows. In Section 2 we present the model problem, followed by the domain decomposition in Section 3. In Section 4, we describe the finite element discretization and the algebraic problem. Results are presented in Section 5.

\section{Model description}

Let $\Omega$ be an open and bounded domain in $\mathbb{R}^{2}$ with smooth boundary $\Gamma=$ $\Gamma_{D} \cup \Gamma_{N}$, where $\Gamma_{D}$ and $\Gamma_{N}$ are Dirichlet and Neumann parts $\left(\Gamma_{D} \cap \Gamma_{N}=\emptyset\right)$. We denote by $\boldsymbol{u}$ the displacement fields and by $\boldsymbol{f}$ the density of the external

\footnotetext{
1 The Lyon University, Universit Jean Monnet Saint-tienne, CNRS UMR 5208, Institut Camille Jordan, F-42023 Saint-Etienne, France, e-mail: olivier.bodart@ univ-st-etienne.fr.$^{2}$ LIMOS, Université Clermont-Auvergne - CNRS UMR 6158, F63000 Clermont-Ferrand, France, e-mail: chorfi@isima.fr, koko@isima.fr
} 
forces. The Cauchy stress tensor $\sigma(\boldsymbol{u})$ and the strain tensor $\epsilon(\boldsymbol{u})$ are given by

$$
\sigma(\boldsymbol{u})=2 \mu \varepsilon(\boldsymbol{u})+\lambda(\varepsilon(u)) \mathbb{I}_{\mathbb{R}^{2}} \quad \text { and } \quad \varepsilon(\boldsymbol{u})=\left(\nabla \boldsymbol{u}+\nabla \boldsymbol{u}^{\top}\right) / 2,
$$

where $\lambda$ and $\mu$ are the Lamé constants. The top boundary ( $\Gamma_{N}$ ground surface) is subject to homogeneous Neumann boundary condition and, on $\Gamma_{D}$ homogeneous Dirichlet boundary conditions are assumed.

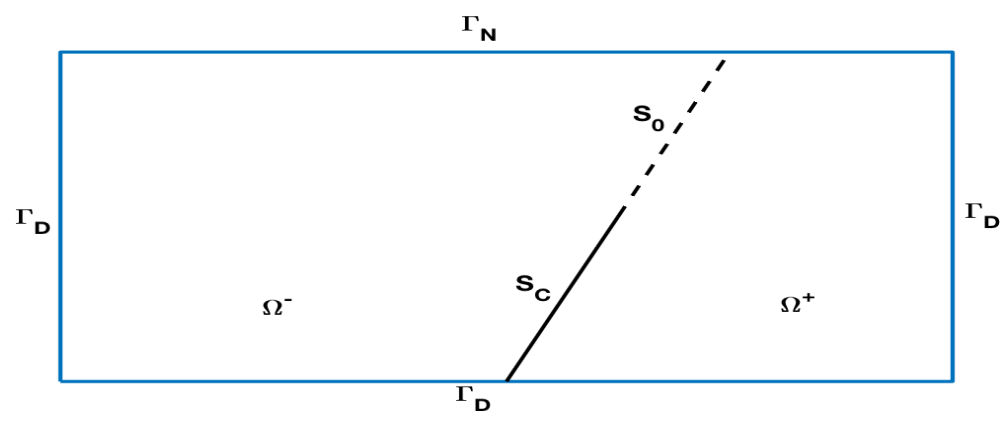

Fig. 1 Domain $\Omega$ with the crack $S_{C}$ and fictitious crack $S_{0}$

We assume that $\Omega$ contains a crack $S_{C}$ represented by a curve (cf. Figure 1), parametrized by an injective map. A nonpenetration condition is prescribed on $S_{C}$. Denoting by $S_{C}^{+}, S_{C}^{-}$the right and left sides of $S_{C}$ we can set $\boldsymbol{u}^{+}=\boldsymbol{u}_{\mid S_{C}^{+}}$and $\boldsymbol{u}^{-}=\boldsymbol{u}_{\mid S_{C}^{-}}$, the displacement fields on the right and left sides of $S_{C}$. Then the nonpenetration condition is given by the negative relative normal displacement, i.e., $\left[\boldsymbol{u}_{n}\right]:=\left(\boldsymbol{u}^{+}-\boldsymbol{u}^{-}\right) \cdot \boldsymbol{n} \leq 0$, assuming no normal gap in the undeformed configuration.

The linear elastostatic model with crack is governed by the following system of equations

$$
\begin{aligned}
-\operatorname{div} \sigma(\boldsymbol{u}) & =\boldsymbol{f} \text { in } \Omega, \\
\boldsymbol{u}=0 \text { on } \Gamma_{D}, \quad \sigma(\boldsymbol{u}) \cdot n & =0 \text { on } \Gamma_{N} . \\
{\left[\boldsymbol{u}_{n}\right] } & \leq 0, \text { on } S_{C} .
\end{aligned}
$$

In the next section we extend the crack to split the domain into two subdomains.

\section{Domain Decomposition}

We extend artificially the crack to split the domain into two subdmoains $\Omega^{ \pm}$ as shown in Figure 1. Let $S_{0}$ be the fictitious crack. On $S_{0}$ we prescribed the 
(displacement) continuity condition $[\boldsymbol{u}]:=\left(\boldsymbol{u}^{+}-\boldsymbol{u}^{-}\right)=0$ and the normal derivative continuity condition $[\sigma(\boldsymbol{u}) n]:=\left(\sigma\left(\boldsymbol{u}^{+}\right)-\sigma\left(\boldsymbol{u}^{-}\right)\right) \cdot n=0$.

Let us introduce the functions space $V=\left\{v \in H^{1}(\Omega) ; v=0\right.$ on $\left.\Gamma_{D}\right\}$, and the forms

$$
a(\boldsymbol{u}, \boldsymbol{v})=\int_{\Omega} \sigma(\boldsymbol{u}): \varepsilon(\boldsymbol{v}) d x \text { and } \boldsymbol{f}(\boldsymbol{v})=\int_{\Omega} \boldsymbol{f} \boldsymbol{v} d x
$$

Then the total potential energy is

$$
J(\boldsymbol{v})=\frac{1}{2} a(\boldsymbol{v}, \boldsymbol{v})-\boldsymbol{f}(\boldsymbol{v}) .
$$

The elastostatic problem with extended crack can now be formulated as the following constrained minimization problem

$$
\begin{aligned}
& \min J(\boldsymbol{u}), \\
& {\left[\boldsymbol{u}_{n}\right] \leq 0 \text { on } S_{C},} \\
& {[\boldsymbol{u}]=0 \text { on } S_{0}}
\end{aligned}
$$

Since the functional (4) is strongly convex on V and constraints (6)-(7) are linear, the constrained minimization problem (5)-(7) has a unique solution.

Remark 1. The stress continuity condition is no longer taken into acount in the formulation (5)-(7). It will be ensure by the Lagrange multiplier associated with the displacement continuity condition (7).

With (5)-(7) we associate the Lagrangian functional $\mathcal{L}$ defined on $\mathbf{V} \times$ $\mathbf{L}^{2}\left(S_{C}\right) \times \mathbf{L}^{2}\left(S_{0}\right)^{2}$ by:

$$
\mathcal{L}\left(\boldsymbol{v}, \mu_{C}, \mu_{0}\right)=J(\boldsymbol{v})+\left(\mu_{C},\left[\boldsymbol{u}_{n}\right]\right)_{S_{C}}+\left(\mu_{0},[\boldsymbol{u}]\right)_{S_{0}},
$$

where $\mu_{C} \in \mathbf{L}^{2}\left(S_{C}\right), \mu_{0} \in \mathbf{L}^{2}\left(S_{0}\right)^{2}$ are the Lagrange multipliers associated with (6) and (7), respectively . Note that the the multiplier associated with (7) must be non negative, i.e. $\mu_{C} \geq 0$ on $S$. Since (5)-(7) is linear a constrained convex minimization problem, a saddle point of $\mathcal{L}$ exists and (5)-(7) is equivalent to the saddle point problem

Find $\left(\boldsymbol{u}, \lambda_{C}, \lambda_{0}\right)$ such that

$$
\mathcal{L}\left(\boldsymbol{u}, \mu_{C}, \mu_{0}\right) \leq \mathcal{L}\left(\boldsymbol{u}, \lambda_{C}, \lambda_{0}\right) \leq \mathcal{L}\left(\boldsymbol{v}, \lambda_{C}, \lambda_{0}\right), \quad \forall\left(\boldsymbol{v}, \mu_{C}, \mu_{0}\right)
$$

Since $\mathcal{L}$ is Gateaux differentiable on $\mathbf{V} \times \mathbf{L}^{2}\left(S_{C}\right) \times \mathbf{L}^{2}\left(S_{0}\right)^{2}$, the solution of (9) is characterized by the saddle-point (Euler-Lagrange) equations of the primal and dual problems as follows

Find $\left(\boldsymbol{u}, \lambda_{C}, \lambda_{0}\right)$ such that 


$$
\begin{aligned}
a(\boldsymbol{u}, \boldsymbol{v})+\left(\lambda_{C},\left[\boldsymbol{v}_{n}\right]\right)_{S_{C}}+\left(\lambda_{0},[\boldsymbol{v}]\right)_{S_{0}} & =\boldsymbol{f}(\boldsymbol{v}), \quad \forall \boldsymbol{v} \in \mathbf{V}, \\
\lambda_{C}\left[\boldsymbol{u}_{n}\right] & =0, \quad \text { on } S_{C}, \\
\left(\mu_{0},[\boldsymbol{u}]\right)_{S_{0}} & =0, \quad \forall \mu_{0} \in \mathbf{L}^{2}\left(S_{0}\right)^{2},
\end{aligned}
$$

where $(.,)_{S_{c}}$ and $(., .)_{S_{0}}$ are $L^{2}$-scalar product on $S_{c}$ and $S_{0}$, respectively. The equality (11) (i.e. the complementarity condition) is true almost everywhere, and if $\lambda_{C}>0$ then $\left[\boldsymbol{u}_{n}\right]=0$, and if $\left[\boldsymbol{u}_{n}\right]<0$ (non contact), then $\lambda_{C}=0$.

\section{Finite element discretization and the algebraic problem}

\subsection{Finite element discretization}

The saddle-point equations are suitable for a fictitious domain approach, i.e. the crack mesh is defined independently of the domain mesh, see e.g.,[6]. We use a fictitious domain method inspired by the extended finite element method (XFEM) in which basis functions are cut across the crack, e.g. [5].

We assume that the domain $\Omega$ has a polygonal shape such that it can be entirely triangulated. Let $\mathcal{T}_{h}$ be a triangulation of $\Omega$.We define the finite elements space

$$
V_{h}=\left\{\boldsymbol{v}_{h} \in \mathcal{C}^{0}(\bar{\Omega}) ; \boldsymbol{v}_{h \mid T} \in P_{k}(T) \forall T \in \mathcal{T}_{h} ; \boldsymbol{v}_{h}=0 \text { on } \Gamma\right\} \subset V,
$$

Here, $P_{k}(T)$ is the space of the polynomials of degree $\leq k$ on the mesh $T$. We define on $S=S_{C} \cup S_{0}$ a finite elements space

$$
\Lambda_{h}=\left\{\lambda^{h} \in \mathcal{C}^{0}(S) ; \lambda_{h \mid I} \in P_{k}(I) \forall I \in \mathcal{I}_{h}\right\} \subset L^{2}(S),
$$

This approach is similar to XFEM [4], except that the standard basis functions near the crack are not enriched by singular functions but only multiplied by Heaviside functions :

$$
H(x)=\left\{\begin{array}{l}
1 \text { if } \\
0 \text { otherwise. }
\end{array}\right.
$$

For element $K$ containing the crack, the stiffness term $\int_{K} \sigma\left(\phi_{i}\right): \varepsilon\left(\phi_{j}\right)$ is replaced by $\int_{K} \sigma\left(H\left(\phi_{i}\right)\right): \varepsilon\left(H\left(\phi_{j}\right)\right)$. 


\subsection{Algebraic problem and algorithm}

Assuming that $\mathbf{u}=\left[\mathbf{u}^{+} \mathbf{u}^{-}\right]^{\top} \in \mathbb{R}^{2 n}$ is the unknown vector of nodal values of the displacement fields on $\Omega_{h}$. Let us define the following matrices and vectors:

- A the stiffness matrix ( $2 n \times 2 n$ symmetric positive definite $), \mathbf{A}=\operatorname{diag}\left(\mathbf{A}^{+}, \mathbf{A}^{-}\right)$.

- $\mathbf{f}$, the external forces (vector of $\mathbb{R}^{2 n}$ ), $\mathbf{f}=\left[\mathbf{f}^{+} \mathbf{f}^{-}\right]^{\top}$

- $\mathbf{B}_{C}$, the relative normal displacement matrix at the contact nodes $\mathbf{B}_{C} \mathbf{u}:=$ $\left(\mathbf{u}^{+}-\mathbf{u}^{-}\right) \cdot \mathbf{n}$.

- $\mathbf{B}_{0}$, the displacement jump matrix across $S_{0}, \mathbf{B}_{0} \mathbf{u}:=\left(\mathbf{u}^{+}-\mathbf{u}^{-}\right)$.

We define the scalar products

$$
(\boldsymbol{\lambda}, \boldsymbol{\mu})_{M_{C}}=\boldsymbol{\lambda}^{\top} M_{C} \boldsymbol{\mu} \text { and }(\boldsymbol{\lambda}, \boldsymbol{\mu})_{M_{0}}=\boldsymbol{\lambda}^{\top} M_{0} \boldsymbol{\mu},
$$

where $M_{C}$ and $M_{0}$ are the mass matrices on $S_{C}$ and $S_{0}$, respectively.

With the above notations, the algebraic Lagrangian of the problem is

$$
\mathcal{L}\left(\mathbf{u}, \boldsymbol{\lambda}_{C}, \boldsymbol{\lambda}_{0}\right)=\frac{1}{2} \mathbf{v}^{\top} \mathbf{A} \mathbf{v}-\mathbf{v}^{\top} \mathbf{f}+\left(\boldsymbol{\lambda}_{C}, \mathbf{B}_{C} \mathbf{v}\right)_{M_{C}}+\left(\boldsymbol{\lambda}_{0}, \mathbf{B}_{0} \mathbf{v}\right)_{M_{0}},
$$

for which the saddle point (KKT) equation are

Find $\left(\mathbf{u}, \boldsymbol{\lambda}_{\boldsymbol{C}}, \boldsymbol{\lambda}_{\mathbf{0}}\right)$ such that:

$$
\begin{aligned}
\nabla_{\mathbf{u}} \mathcal{L}\left(\mathbf{u}, \boldsymbol{\lambda}_{C}, \boldsymbol{\lambda}_{0}\right) & =0 \\
\nabla_{\boldsymbol{\lambda}_{C}} \mathcal{L}\left(\mathbf{u}, \boldsymbol{\lambda}_{C}, \boldsymbol{\lambda}_{0}\right) \leq 0, \quad \boldsymbol{\lambda}_{C} \geq 0, \boldsymbol{\lambda}_{C} \cdot \nabla_{\boldsymbol{\lambda}_{C}} \mathcal{L}\left(\mathbf{u}, \boldsymbol{\lambda}_{C}, \boldsymbol{\lambda}_{0}\right) & =0 \\
\nabla_{\boldsymbol{\lambda}_{0}} \mathcal{L}\left(\mathbf{u}, \boldsymbol{\lambda}_{C}, \boldsymbol{\mu}_{0}\right) & =0,
\end{aligned}
$$

where $(\cdot)$ stands for element-wise (or Hadamard) multiplication. Note that in (13), the primal problem, the unknowns $\mathbf{u}^{ \pm}$are uncoupled if the Lagrange multipliers $\left.\boldsymbol{\lambda}_{C}, \boldsymbol{\lambda}_{0}\right)$ are known. Then a primal-dual algorithm is suitable for solving (13)-(15). To apply an primal-dual interior point method, we set $\mathbf{z}=-\nabla_{\boldsymbol{\lambda}_{C}} \mathcal{L}\left(\mathbf{u}, \boldsymbol{\lambda}_{C}, \boldsymbol{\lambda}_{0}\right)$, such that (13)-(15) becomes

Find $\left(\mathbf{u}, \mathbf{z}, \boldsymbol{\lambda}_{C}, \boldsymbol{\lambda}_{0}\right)$, with $\mathbf{z} \geq 0$ and $\boldsymbol{\lambda}_{C} \geq 0$, such that

$$
\begin{aligned}
\nabla_{\mathbf{u}} \mathcal{L}\left(\mathbf{u}, \boldsymbol{\lambda}_{C}, \boldsymbol{\lambda}_{0}\right) & =0 \\
\nabla_{\boldsymbol{\lambda}_{C}} \mathcal{L}\left(\mathbf{u}, \boldsymbol{\lambda}_{C}, \boldsymbol{\lambda}_{0}\right)+\mathbf{z} & =0, \\
\nabla_{\boldsymbol{\lambda}_{0}} \mathcal{L}\left(\mathbf{u}, \boldsymbol{\lambda}_{C}, \boldsymbol{\lambda}_{0}\right) & =0 . \\
\boldsymbol{\lambda}_{C} \cdot \mathbf{z} & =0
\end{aligned}
$$

Since $\mathbf{A}$ is positive definite, (16)-(19) are necessary and sufficient conditions. Then we have to solve a nonlinear system of the form 


$$
F\left(\mathbf{u}, \mathbf{z}, \boldsymbol{\lambda}_{C}, \boldsymbol{\lambda}_{0}\right)=0, \quad \mathbf{z} \geq 0, \quad \boldsymbol{\lambda}_{C} \geq 0 .
$$

Let us introduce the vector $\mathbf{e}=(1, \ldots, 1)^{\top}$ and define the complementarity measure $\mu=\boldsymbol{\lambda}_{C}^{\top} \mathbf{z} / m$, where $m$ is the dimension of $\mathbf{z}$. We then replace (20) by the following perturbed KKT conditions

$$
F\left(\mathbf{u}, \mathbf{z}, \boldsymbol{\lambda}_{C}, \boldsymbol{\lambda}_{0}\right)=\left(0^{\top}, 0^{\top}, 0^{\top}, \tau \mu \mathbf{e}^{\top}\right)^{\top},
$$

that is

$$
\begin{aligned}
\mathbf{A} \mathbf{u} & =\mathbf{f}-B_{C}^{\top} \boldsymbol{\lambda}_{C}-B_{0}^{\top} \boldsymbol{\lambda}_{0}, \\
\mathbf{B}_{C} \mathbf{u}+\mathbf{z} & =0, \\
\mathbf{B}_{0} \mathbf{u} & =0, \\
\boldsymbol{\lambda}_{C} \cdot \mathbf{z} & =\tau \mu \mathbf{e},
\end{aligned}
$$

where $(\tau, \mu)>0$. Solutions of (22)-(25) for all positive values of $\tau$ and $\mu$ define a curve $\mathcal{C}(\tau, \mu)$, called the central path, which is the trajectory that leads to the solution of the quadratic problem as $\tau \mu$ tends to zero. The primaldual interior point algorithm for solving the saddle point system (13)-(15) consists of applying the damped Newton method to (22)-(25). The damped parameter, $\tau$ and $\mu$ are adjusted iteratively to ensure fast convergence (see e.g., [3, sect 16.6],[7]). Solving (21) with primal-dual interior point method consists of solving a primal-dual linear system equivalent to the optimality conditions for an equality-constrained convex quadratic program. Applying a Uzawa conjugate gradient method to the (linearized) optimality conditions

leads to solving primal linear systems of the form (22) which breaks down naturally into \pm sub-systems.

\section{Numerical results}

We have implemented the method described in the previous section in MATLAB (R2016b) on a Linux workstation equipped with a quad-core Intel Xeon E5 with 3.00GHz clock frequency and 32GB RAM. We use the mesh generation package KMG2D [1], and the fast FEM assembling functions package KPDE [2]. The test problem used is designed to illustrate the numerical behavior of the algorithm more than to model an actual geological crack.

We consider $\Omega=(0,10) \times(0,5)$ with the boundary partition

$$
\begin{aligned}
& \Gamma_{D}=(0,10) \times\{0\} \cup\{1\} \times(0,5) \cup\{0\} \times(0,5) \\
& \Gamma_{N}=(0,10) \times\{1\} .
\end{aligned}
$$

The crack is given by 
$S_{C}=\left\{(x, 1.25(x-3) \mid x \in(3,5.4)\}, \quad S_{0}=\{(x, 1.25(x-3) \mid x \in(5.4,7)\}\right.$.

The mesh sample is shown in Figure 2. The material constants are $E=9 \times 10^{6}$

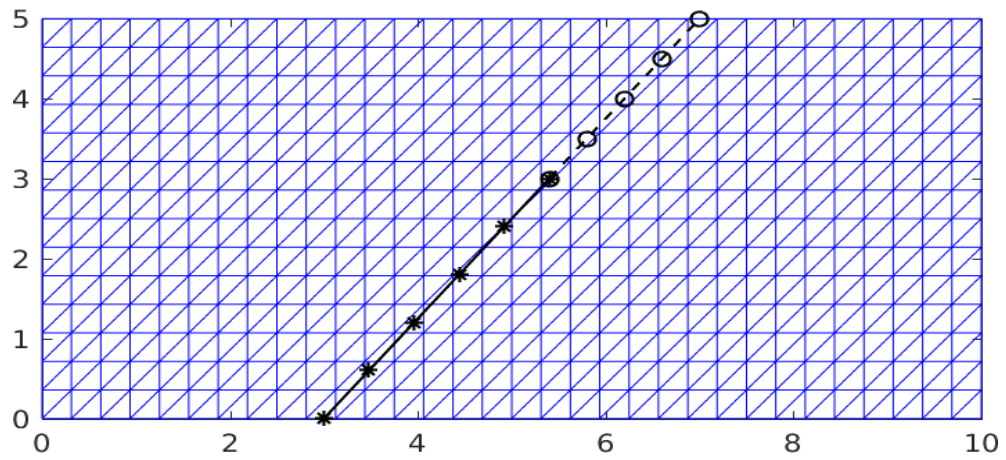

Fig. 2 Mesh sample of $\Omega$ with real crack and fictitious crack

(Young's modulus) and $\nu=0.3$ (Poisson's ratio). The applied force to the domain is the gravity with a value density of 1500 .

We use the couple $P 2 / P 1$ for the discretization: continuous $P 2$ triangular element for $\Omega_{h}$, continuous $P 1$ segment for the crack. The choice of the finite element pair $P 2 / P 1$ is made to ensure the inf-sup condition. We first consider a uniform discretization of $\Omega$ consisting of 561 nodes and 256 triangles. The interior point algorithm stops after 11 iterations. The deformed configuration is shown in Figure 3 and reveals the presence of a crack.

To study the behavior of our algorithm, the initial mesh is uniformly refined to produce meshes with 2145, 8385, 33153 and 131841 nodes. The performances of the algorithm is shown in Table 1. One can observe that the number of iterations required for convergence is virtually independent of the mesh size.

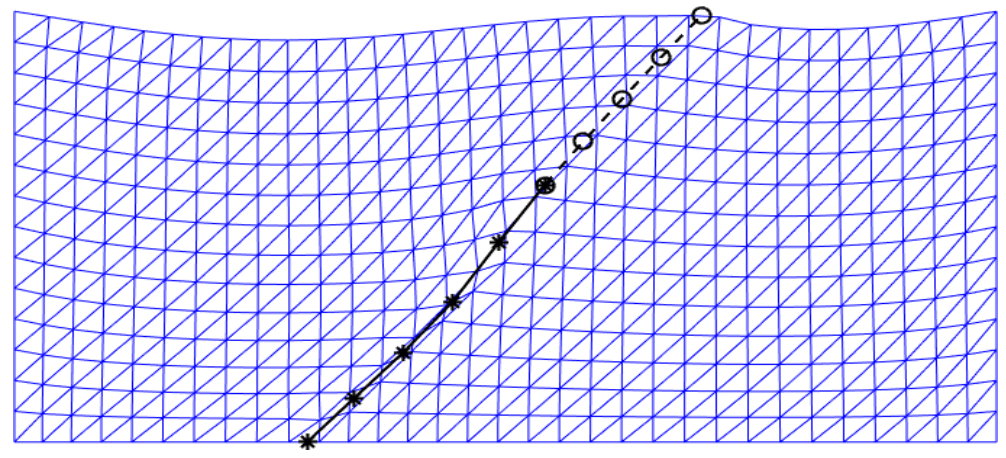

Fig. 3 Mesh sample of $\Omega$ with real crack and fictitious crack (magnification=20) 


\begin{tabular}{|c||c|c|c|c|c|}
\hline Nodes/Triangles & $561 / 256$ & $2145 / 1024$ & $8385 / 4096$ & $33153 / 16384$ & $131841 / 65536$ \\
\hline Iterations & 11 & 12 & 15 & 15 & 14 \\
CPU Times (Sec.) & 0.21 & 0.47 & 2.37 & 17.64 & 191.92 \\
\hline
\end{tabular}

Table 1 Number of iterations and CPU times (in Sec.) for the interior point algorithm

\section{Conclusion}

We have studied a fictitious domain method for a geological crack based on fictitious domain and XFEM. Numerical experiments show that the number of iterations is virtually independent of the mesh size. Further work is under way to accelerate the method using preconditioning techniques inspired by [7]. Stabilization techniques, as in [5], are also under study.

\section{References}

[1] Koko J. A MATLAB mesh generator for the two-dimensional finite element method. Appl. Math. Comput., 250:650-664, 2015.

[2] Koko J. Fast MATLAB assembly of fem matrices in 2d and 3d using cell array approach. Int. J. Model. Simul. Sci. Comput., 7, 2016.

[3] Nocedal J. and Wright S.J. Numerical Optimization. Springer, 2006.

[4] Moës N., Dolbow J., and Belytschko T. A finite element method for crack growth without remeshing. Int. J. Numerical Mathods Engineering, 46:131-150, 1999.

[5] Bodart O., Cayol V., Court S., and Koko J. Xfem-based fictitious domain method for linear elastic model with crack. SIAM J. Sci. Comput., 38:219-246, 2016.

[6] Glowinski R., Pan T.W., and Periaux J. A fictitious domain method for dirichlet problem and applications. Comput. Methods Appl. Mech. Engrg., 111:283-303, 1994.

[7] Kučera R., Machalová J., Netuka H., and Zenčá P. An interior-point algorithm for the minimization arising from 3d contact problems with friction. Optimization Methods and Software, 28:1195-1217, 2012. 